\title{
Influence of Length Variation in Bamboo Fiber on Tensile Strength and Compressive Strength of Concrete
}

\author{
R Trimurtiningrum ${ }^{1}$, L Endah ${ }^{2}$, F Aziz $^{3}$ \\ $\left\{\right.$ nenos47@yahoo.com $\left.{ }^{1}\right\}$ \\ ${ }^{1,2,3}$ Civil Engineering,Universitas 17 Agustus 1945 Surabaya, Indonesia
}

\begin{abstract}
Concrete is one of the most commonly used building construction materials since concrete has advantages such as high compressive strength, can be molded to any desired shape and easy to find material for a relatively cheap price. However, concrete also has disadvantages such as brittle and has low tensile strength. Tensile strength of concrete is only about $8-10 \%$ of its compressive strength. From previous researches, the use of fibers is effective to increase the tensile strength of concrete. Types of fiber that commonly use in concrete mixtures are natural fibers from animals and plants, also artificial fibers such as steel, glass fibers, and synthetic fibers. Natural fiber concrete such as bamboo has low manufacturing cost than the other types of fiber. The aim of this research is to investigate the influence of fiber size in bamboo fiber on tensile strength and compressive strength of concrete. The percentage of bamboo fiber addition in concrete mixtures was $2 \%$ with variation of fiber $2 \mathrm{~cm}, 3 \mathrm{~cm}$ and 4 $\mathrm{cm}$ in length. The optimum tensile strength and compressive strength of the concrete is obtained from bamboo fiber concrete with $2 \mathrm{~cm}$ variation in length, which is $107,41 \mathrm{~kg} / \mathrm{cm} 2$ and $230,03 \mathrm{~kg} / \mathrm{cm} 2$ respectively at 28 days.
\end{abstract}

Keywords:Fiber Concrete, Bamboo Fiber, Tensile Strength, Compressive Strength

\section{Introduction}

Concrete is construction materials that have been used widely almost in every country. The advantages of using concrete as construction material are it can be molded to any desired shape, easy to find material for relatively low price and has high compressive strength. However, concrete has a low tensile strength. Concrete is brittle material and has tensile strength for about $8-10 \%$ from its compressive strength. Brittle material usually has limited ductility and low resistance to crack. Cracks in concrete must be controlled because cracks caused the entrance of water and another aggressive mineral into concrete structure and caused corrosion to the steel reinforcement. From previous research, the use of fibers is effective to increase the tensile strength of concrete.

Fiber concrete is concrete containing fiber which is uniformly distributed and randomly oriented in the cement matrix. Fiber concrete is being investigated to enhance the tensile 
strength of concrete and to inhibit the growth of tensile cracks in concrete [1]. In recent years, the concept of fiber reinforced concrete is a great development to enhance the performance of concrete such as high strength and durability of concrete [2].There is numerous type of fibers, such as synthetic fiber like steel, glass, and carbon also natural fiber materials from plants and animals that have been examined to make fiber concrete. The manufacture of synthetic fiber is quite expensive and consume considerable energy [3]. Therefore, natural fiber like bamboo becomes a solution.

For a long period, bamboo has been known as one of the oldest building material because it characterized by high strength and low weight, and is easily worked using simple tools [4]. Bamboo as building material has been used for the construction of scaffolding, bridges, and house. Bamboo plant growth well and fast also has minimum energy to harvest and transport. Therefore bamboo has low manufacturing cost compared with synthetic fiber such as glass or steel fibers [5].

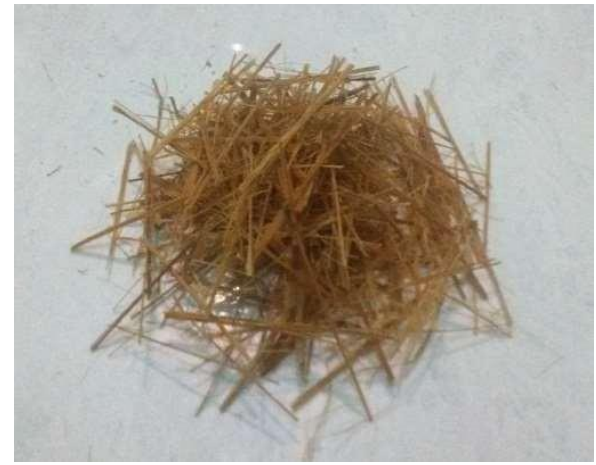

Fig1. BambooFiber

Theaimofthisresearchistoinvestigatetheinfluence freshandhardenedstateofconcrete, suchasworkability, offibersizeinbamboofiberon strengthofconcrete. tensilestrengthandcompressive

\section{Method}

\subsection{Materials}

CementThisresearchusePortlandcementtypeIfromPT.SemenGresik,Indonesia.

\subsection{Fine Aggregates}

FineaggregateswerefromnaturalriversandfromEastJawa,Indonesia.Fromt h e sieveanalysis result,thisresearchusingfineaggregatewith gradingzone 2 . 


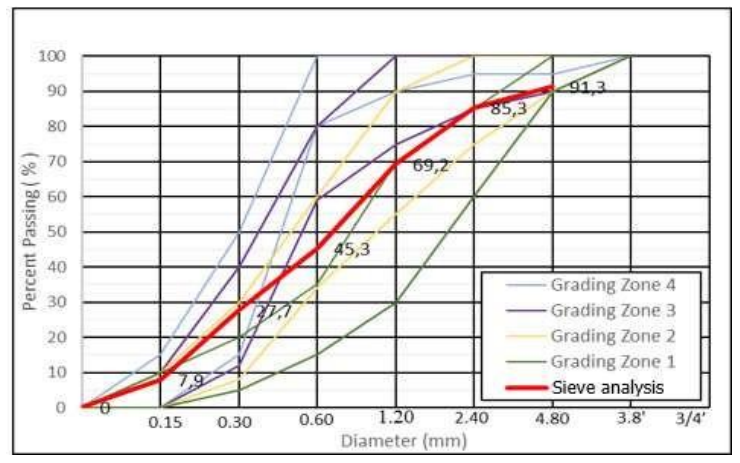

Fig 2. Sieve analysis result of fine aggregate

\subsection{Coarse Aggregates}

Coarseaggregateswerecrushedgravelwith40-mmmaximumdiameter.Thesieveanalysisresult fromcoarseaggregatesisshowninfig.3.

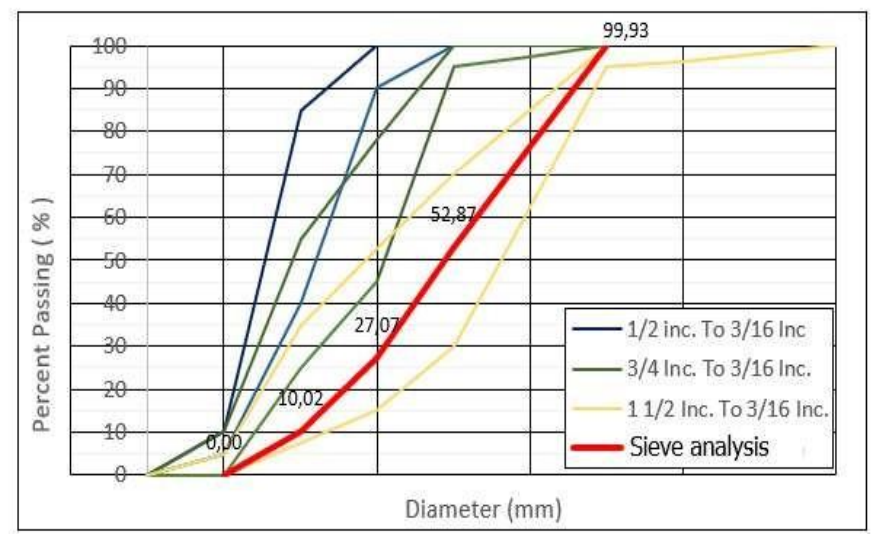

Fig3

Sieveanalysisresultofcoarseaggr

egate

Physicalpropertiesoffineandcoarseaggregatesarereportedintable1.

Table1.Physicalpropertiesoffineandcoarseaggregates

\begin{tabular}{ccc}
\hline Physicalproperties & San & Crushedgravel \\
\hline Moisturecontent & $2.72 \%$ & 0,42 \\
\hline Specificgravity & 2.6 & 2.6 \\
\hline Waterabsorption & $1.43 \%$ & 2.49 \\
\hline Bulkdensity & $1490 \mathrm{~kg} / \mathrm{m} 3$ & $1230 \mathrm{~kg} / \mathrm{m}^{3}$ \\
\hline
\end{tabular}

\subsection{Bamboo Fiber}

ThisresearchusingoriginalbamboofromEastJawa,Indonesia. 


\subsection{Experimental Procedure}

Mixtureproportions:

Therewere4mixturespreparedforthisresearch.Onemixture ascontrolled specimen whichhas $0 \%$ ofbamboofiber.Theother mixturescontain bamboofiber forabout $2 \%$ weightof cementwitha variationoffiber $2 \mathrm{~cm}, 3 \mathrm{cmand} 4 \mathrm{cminlength}$.

Mixturesproportionwerecalculatedusingthe DOEmethodwith $200 \mathrm{~kg} / \mathrm{cm}^{2}$ compressive strength. Mixtureproportionsofconcreteareshownintable2.

Table2. ConcreteMixturesforcompressivestrength $200 \mathrm{~kg} / \mathrm{cm}^{2}$ pereachm ${ }^{3}$

\begin{tabular}{|c|c|c|c|c|c|c|c|}
\hline \multirow[t]{2}{*}{ Mixtures } & \multirow[t]{2}{*}{$\begin{array}{c}\text { Cement } \\
(\mathrm{kg})\end{array}$} & \multirow[t]{2}{*}{$\begin{array}{l}\text { Water } \\
(\mathbf{k g})\end{array}$} & \multirow[t]{2}{*}{$\begin{array}{c}\text { Fine } \\
\text { Aggreggate } \\
\text { (kg) }\end{array}$} & \multirow[t]{2}{*}{$\begin{array}{c}\text { Coarse } \\
\text { Aggreggate } \\
\text { (kg) }\end{array}$} & \multicolumn{3}{|c|}{$\begin{array}{c}\text { Fiber } \\
\text { (2\% weight } \\
\text { of cement) } \\
(\mathrm{kg})\end{array}$} \\
\hline & & & & & $2 \mathrm{~cm}$ & $3 \mathrm{~cm}$ & $4 \mathrm{~cm}$ \\
\hline $\mathrm{NC}$ & 308,33 & 199,39 & 747,28 & 1130 & - & - & - \\
\hline FC-2 & 308,33 & 199,39 & 747,28 & 1130,01 & 6,17 & - & - \\
\hline FC-3 & 308,33 & 199,39 & 747,28 & 1130,01 & - & 6,17 & - \\
\hline FC-4 & 308,33 & 199,39 & 747,28 & 1130,01 & - & - & 6,17 \\
\hline
\end{tabular}

Nameofmixturesexplanation:

$\mathrm{NC}$

$=$ Normalconcrete $($ controlledspeci

men) FC-X,defines:

FC $=$ Fiberconcrete

$\mathrm{X} \quad$ =Lengthoffiberin $\mathrm{cm}$

SometestingmethodthatusedinthisresearchwereslumptestaccordingtoASTMC143 [6],compressive strengthtestaccordingtoASTMC39[7]andsplittingtensilestrengthtest according toASTMC496[8].Both,compressive strengthtestandsplittestusingspecimensof concretethatwerecylindrical with $15 \mathrm{cmindiameterand} 30 \mathrm{cminheightandwerecuredby}$ submergedintothewaterinroomtemperatureasshowninfig4.Thatspecimenweretestedat theageof7and28days.

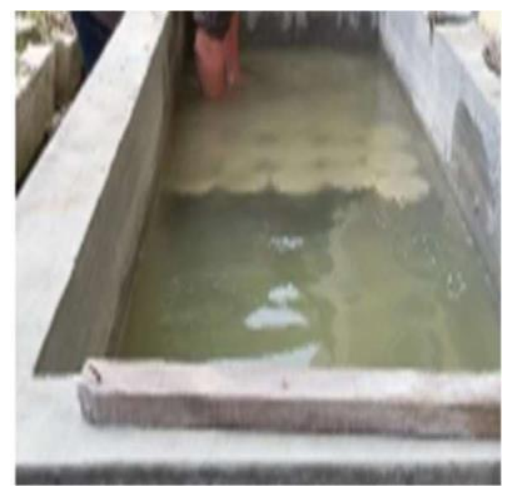

Fig4 Concretecuring 


\section{Result and Discussion}

Slumptestresult.Thistestresultdefinestheworkabilityoffreshconcrete. Fig5showsthe influenceofbamboofiberlengthvariationonslumptestresult.

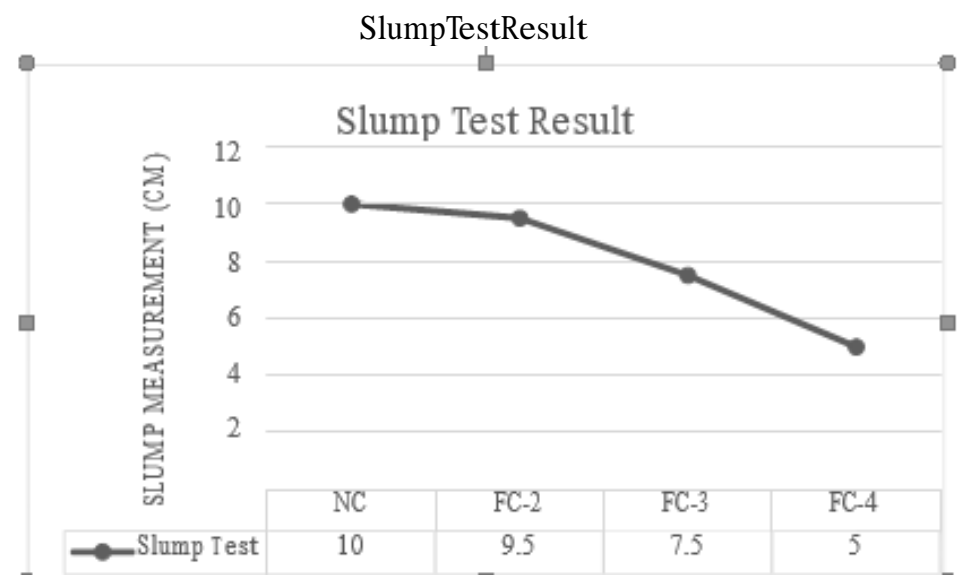

Fig5 Effectofbamboofiberlengthonthe workabilityof freshconcrete

Slump test measurement determines the workability of concrete. Concrete mixtures that have lowervalue of slump measurement means has lower workability. The result shown in fig 5 concluded that the presence of bamboo fiber also the length of bamboo fiber influence the workability of concrete. Normal concrete (NC) which has $0 \%$ of bamboo fiber has the highest slump test result for about $10 \mathrm{~cm}$, however, concrete mixtures which containing bamboo fiber have lower value of slump measurement because of bamboo fiber tend to absorb the free water content in the mixture.

Fig 5 also shown that longer size of fiber results in lower slump test which because longer size of fiber tends to have larger surface area that means more water being absorbed by the bamboo fiber.

Compressive strength test result. Fig 6 shows the difference of crack failure from normal concrete and fiber concrete

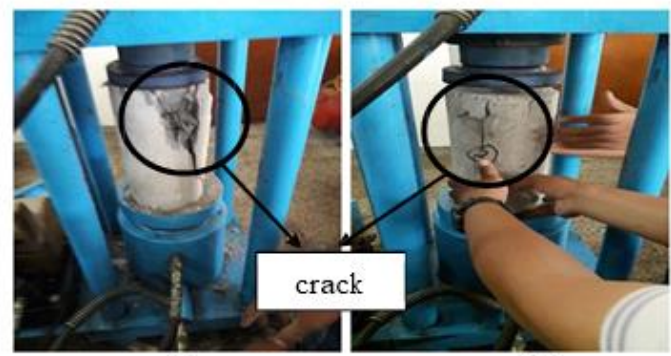

(a)

(b) 
Fig6 (a)Compressivestrengthtestofnormalconcrete(b)Compressivestrengthtestoffiber concrete

Itisshownthatnormal concretehasseverecrackfailure thanfiberconcrete, thatisbecausethe presenceoffibermakeconcretehaslargercrackresistancedueto bindingof fiber[9].Therefore, concretewithfiberhasbettercrackcontrol.

Fig.7showsarelation ofbamboofiber lengthvariationinthemixtureandcompressivestrength ofconcrete.

Fig7 Theinfluenceofbamboofiberlengthonthecompressivestrengthof concrete

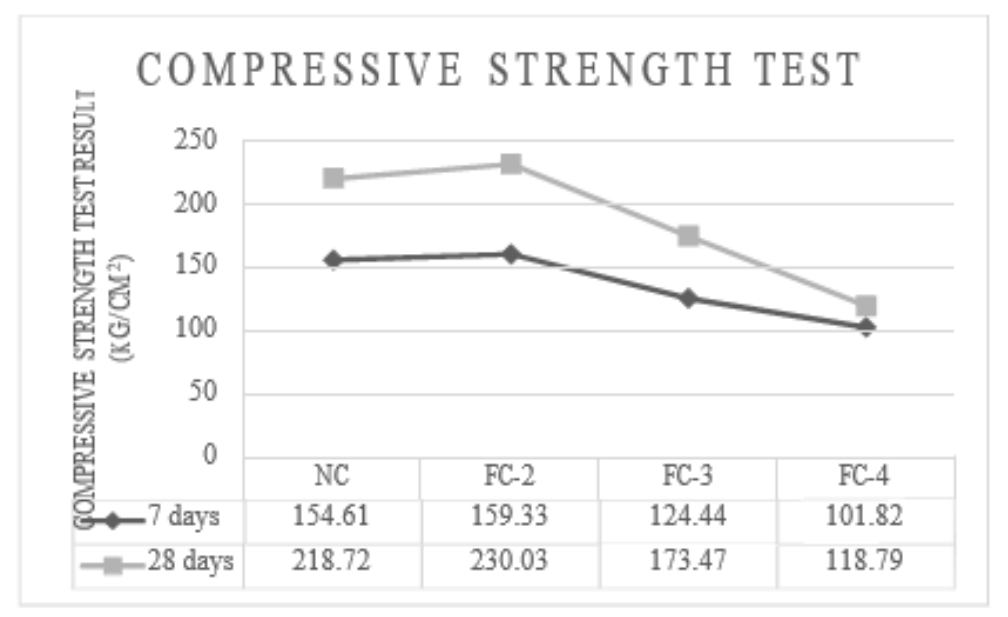

Theresultshowedthatthereisa slightincreaseinthecompressive strengthinconcretewith bamboofiber. Thecompressivestrengthof concretewhichhas $2 \mathrm{~cm}$ lengthofbamboofiber(FC2)is $159,33 \mathrm{~kg} / \mathrm{cm} 2$ at 7 daysand230,03kg/cm2at28days,increase about $3 \%$ and $5 \%$ respectively comparedwithnormalconcrete(NC).FC-3andFC-4whichhavelongerbamboo fibershowlowercompressivestrengthvalue. Thecompressivestrengthofconcretedecreasefor about 19\%-34\%thannormalconcrete.Itisbecausethemixtureshavelessworkabilitywhichmade thecompactionprocessofconcreteharderandincreasesthevolumeporesofconcrete.

Splittingtensilestrengthtestresult.Fig8shows theeffectofbamboo lengthfiberonsplitting strengthtestresult.

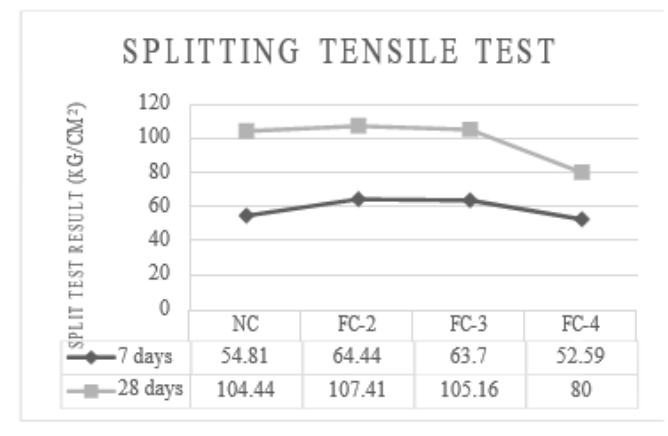




\begin{abstract}
Fig8Influenceofbamboofiberlengthonthesplittingtensilestrengthofconcrete
Itisshownthatthereisincreaseresultimeoncretewith2cmol splittingtensilestrengthtestresultincrease byabout $18 \%$ and $3 \%$ at 7 daysand28daysrespectively thannormalconcrete.Itisbecausethefibersmakeconcretetobemoreresistancetocracksothat theconcreteabletoreceive higher tensileforce.Fiberconcretewhichhaslongerbamboofiber (FC3andFC-4)showlowertensilestrengthvalue.Thatisbecauseofthedecreaseinworkability ofconcretemakethecompactionprocesshardersothatincreasethevolumeporesinconcreteand decreasethebondbetweenconcreteconstituentmaterials.
\end{abstract}

\title{
2 Conclusion
}

Lengthofconcretefiberinfluencetheworkabilityofconcrete, thecompressivestrength andthesplittestresult.ConcretewithfiberhaslessworkabilitythanordinaryPortlandcementconcrete .Longer sizeofbamboofiberhasa lowerslumpvaluetest.Itisbecausebamboofiberabsorbsthefree watercontentinthemixture. A mixturethathas $2 \mathrm{cmlengthoffiberconcretehastheoptimumcompressivestrengthtest}$ andsplittestresult.Itisbecausefiberconcretetendstohavehigherresistancetocrackdue tothefiberbinding.Thereforethespecimenable toreceivehigher axialandtensileforce thannormalconcrete.Bothcompressivestrengthandsplittestresultdecreaseinmixturesthathave $3 \mathrm{~cm}$ and4 cmoffiberlengthbecausethemixtureshavelessworkabilitywhichmadethecompaction processofconcreteharderandincreasethevolumeporesof concrete.

\section{References}

[1] S. M. Dewi, M. N. Wijaya, and C. R. N., "The use of bamboo fiber in reinforced concrete beam to reduce crack," in AIP Conference Proceedings, 2017, p. 20003.

[2] K. V. Sabarish, E. S. E., and A. C., "An Experimental Investigation of Bamboo Fiber in Concrete Elements by Replacement of Fine Aggregate," SSRG Int. J. Ciil Eng., no. Special Issue, pp. 6-8, 2017.

[3] R. Sethunarayanan, S. Chockalingam, and R. Ramanathan, "Natural fiber reinforced concrete," Transp. Res. Rec., vol. 1226, pp. 57-60, 1989.

[4] P. Sharma, K. Dhanwantri, and S. D. K. M. Mehta, "Bamboo as a building material," Int. J. Civ. Eng. Res., vol. 5, no. 3, pp. 249-254, 2014.

[5] M. Terai and K. Minami, "Fracture Behavior and Mechanical Properties of Bamboo Fiber Reinforced Concrete," Key Eng. Mater., vol. 488-489, pp. 214-217, Sep. 2011.

[6] ASTM International, "ASTM C143, Standard Test Method for Slump of HydraulicCement Concrete.” ASTM International, Pennsylvania, 2005.

[7] ASTM C39, "Standard Test Method for Compressive Strength of Cylindrical Concrete Specimens.” ASTM International, Pennsylvania, 2001.

[8] ASTM C496, "Standard Test Method for Splitiing Tensile Strength of Cylindrical Concrete Specimens.” ASTM International, Pennsylvania, 1996.

[9] S. Vajje, "Study on addition of the natural fibers into concrete," Int. J. Sci. Technol. Res., vol. 2, no. 11, pp. 213-218, 2013. 